\title{
Nosocomial pneumonia with multiresistant germs and probabilistic antibiotic therapy in Medical Resuscitation: year 2013-2014
}

\author{
Ezzouine H., Slimani Y., Benslama A. \\ Medical intensive care unit \\ Dept of Anaesthesiology \& Intensive Care, \\ University Hassan II Faculty of Medicine and Pharmacy, \\ Casablanca, Morocco
}

Background and Goal of Study:

Nosocomial pneumonia remains a major complication occurring in an intensive care setting, especially in artificially ventilated patients. Their incidence is constantly increasing, despite the regular appearance of new therapeutics. This is due to changes in patient care and recruitment practices.

It is a retrospective descriptive and analytical study carried out at the Medical intensive care unit of the Ibn Rushd Hospital in Casablanca, spread over a period of 12 months, between January and December 2014.

Materials and Methods:

We analyzed all the bronchial samples taken in the diagnosis of nosocomial pneumonia.The statistical analysis was carried out using SPSS 22.0 and EXCEL 2011.

Results and Discussion:

-Incidence of Nosocomial pneumonia with multiresistant germs :37.9\%.

- The average age of patients was 43.30 years with extremes ranging from 16 to 83 years.

$-66.4 \%$ of the patients were male.

-The most widely prescribed antibiotics for initial probabilistic antibiotic therapy were:

-Amikacin (53\% of prescriptions),

-Imipenem (52\% of prescriptions),

-Colimycin (49\% of prescriptions)

- Rifampicin (38\% \% Of prescriptions).

$-75.7 \%$ of the patients in our series, received antibiotic therapy within 24 hours of admission and $24.3 \%$ after 24 hours.

-The average duration of treatment was 14.3 days.

-Our patients could not benefit from a therapeutic deescalation.

\begin{tabular}{|l|r|r|}
\hline Acinetobacter baumanii & 73 & $17 \%$ \\
\hline Pseudomonas aeruginosa & 42 & $10 \%$ \\
\hline Klebsiella pneumoniae ss. & 23 & $5 \%$ \\
\hline Haemophilus influenzae & 21 & $5 \%$ \\
\hline Streptococcus pneumoniae & 19 & $4 \%$ \\
\hline Staphylococcus aureus ss. Aureus & 19 & $4 \%$ \\
\hline Escherichia coli & 13 & $3 \%$ \\
\hline Yeast & 7 & $2 \%$ \\
\hline
\end{tabular}
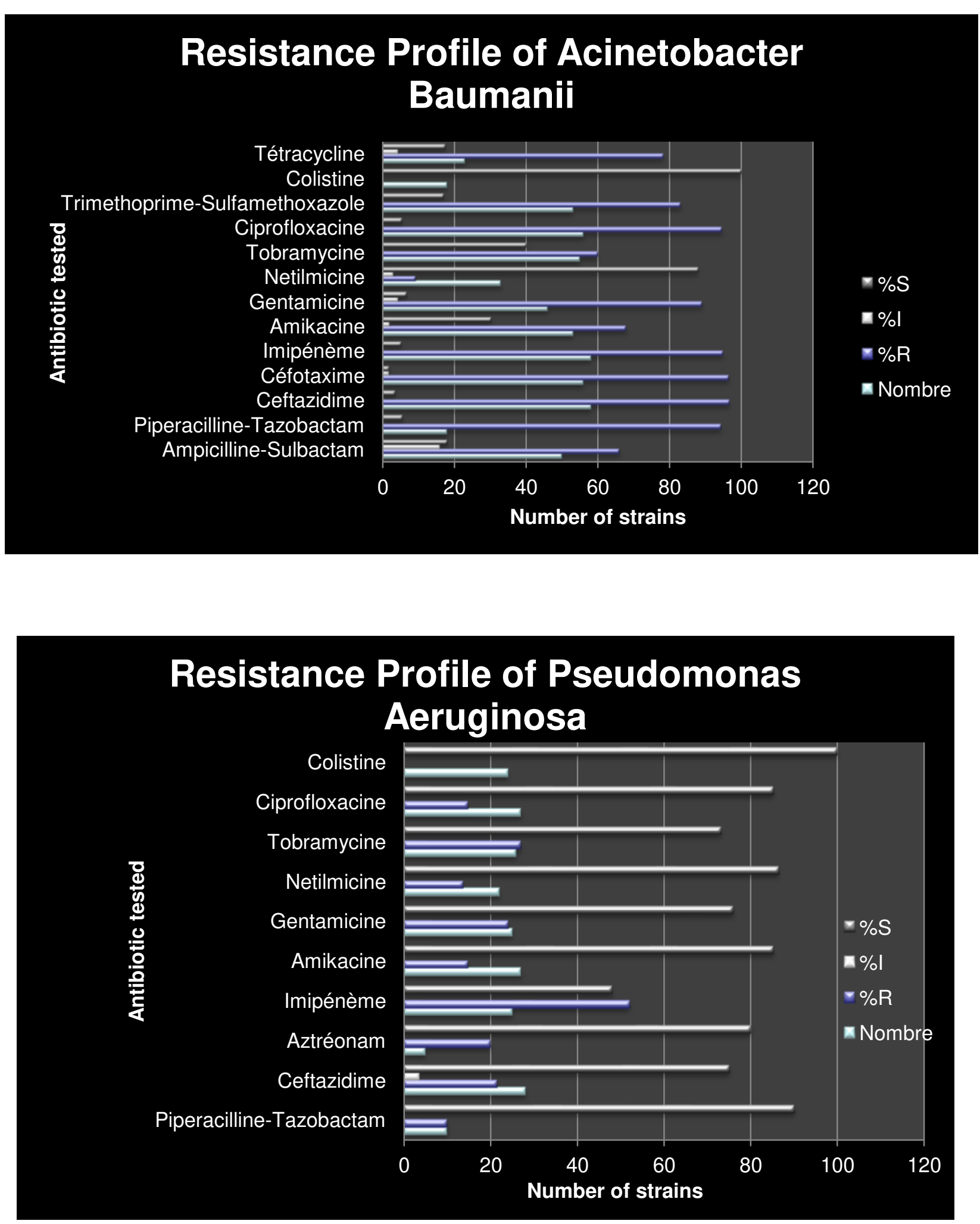

-The factors associated with mortality in intensive care were sought in univariate analysis. A value of $p<0.05$ was considered as the threshold of significance.

-Charlson,APACHE II, SAPS II and SOFA scores, duration of intubation, sedation, the late occurrence of pneumonia were factors correlated with excess mortality.

Conclusion(s):

Nosocomial pneumonia with multiresistant germs and probabilistic antibiotic therapy in Medical Resuscitation are a great dilemma for intensivists in the daily practice. Some data are more correlated to mortality even if a probabilistic antibiotherapy managed earlier for nosocomial pneumonia as Charlson score, APACHEII, SAPS II, SOFA, duration of intubation and sedation and the timing of occurence pneumonia. 